Communication, technologies et développement

$6 \mid 2018$

Technologies mobiles, innovation et développement

\title{
Alain Kiyindou, Noble Akam, Etienne Damome (Dirs), Objets connectés et développement intelligent
}

Paris, L'Harmattan, 2018, Communication et Civilisation, 277 pages

\section{Carole Fagadé}

\section{OpenEdition}

\section{Journals}

Édition électronique

URL : http://journals.openedition.org/ctd/915

DOI : $10.4000 /$ ctd. 915

ISSN : 2491-1437

Éditeur

Chaire Unesco Pratiques émergentes en technologies et communication pour le développement

Référence électronique

Carole Fagadé, «Alain Kiyindou, Noble Akam, Etienne Damome (Dirs), Objets connectés et développement intelligent », Communication, technologies et développement [En ligne], 6 | 2018, mis en ligne le 18 décembre 2018, consulté le 24 septembre 2020. URL : http://journals.openedition.org/ctd/ 915 ; DOI : https://doi.org/10.4000/ctd.915

Ce document a été généré automatiquement le 24 septembre 2020

Communication, technologies et développement 


\title{
Alain Kiyindou, Noble Akam, Etienne Damome (Dirs), Objets connectés et développement intelligent
}

Paris, L'Harmattan, 2018, Communication et Civilisation, 277 pages

\author{
Carole Fagadé
}

\section{RÉFÉRENCE}

Alain Kiyindou, Noble Akam, Etienne Damome (Dirs), Objets connectés et développement intelligent, Paris, L'Harmattan, 2018, Communication et Civilisation, 277 pages.

1 Avec quatorze contributions, "Objets connectés et développement intelligent » est un ouvrage fondamental sur la question de l'utilisation des objets connectés et le processus de développement. A travers des approches transdisciplinaire et interdisciplinaire, cette publication issue des travaux de la Chaire Unesco Pratiques émergentes en technologie et communication pour le développement interroge l'appropriation des objets connectés et leur usage dans des domaines aussi variés que la santé, l'agriculture donnant ainsi lieu à un nouveau concept, le développement intelligent. Cette « intelligence » se traduit par des pratiques de collecte, d'analyse et de diffusion des informations en vue d'une action plus réfléchie et donc plus efficace. L'ouvrage est structuré en deux parties.

2 La première partie, constituée de sept contributions dégage les enjeux des objets connectés tout en questionnant à nouveau le concept de développement. Christelle CAZABAT revient sur la notion de développement humain qu'elle lie à la naissance d'une nouvelle société au sein de laquelle l'individu est placé au coeur des stratégies de développement, et ce conformément aux orientations du Programme des Nations Unies pour le Développement (PNUD). Elle reprend les termes du rapport sur le développement humain de 1990 selon lequelle « le but du développement est d'élargir la gamme des opportunités qui s'offrent aux individus». Se référant à quelques 
initiatives publiques dans le domaine de la santé, l'éducation, l'économie et la politique, elle souligne l'influence des TIC dans la promotion et la participation citoyenne.

Mohamed Ali MAZEGH, Khadidja YOUSSOUFI et Abderrahmane AMSIDDER orientent leur analyse sur la perception des objets connectés au Maroc. Ils font remarquer que la question des objets connectés n'est pas encore au centre des préoccupations des Marocains. Lorsqu'ils sont connus, ils apparaissent comme des symboles de sécurité, de stabilité et d'assurance ou sont aisément assimilés au téléphone mobile largement répandu en Afrique. Gnéré Blama DAGNOGO explique cette appropriation en ce qu'il est avant tout un outil de l'oralité, une des principales caractéristiques des sociétés africaines. Anoman Nathalie DON établit une corrélation entre les objets connectés et l'existence humaine à travers ce qu'il est convenu d'appeler l'homme augmenté. Les TIC permettent ainsi de développer le conatus défini par Spinoza comme "l'effort ou la puissance propre de tout individu à persévérer dans son être ...Cet effort exprime la puissance actuelle de cet être » (Spinoza, 1997, p. 421).

4 Caroline BAYART, Agnès LANCINI et Catherine VIOT, reviennent sur la notion d'appropriation des objets connectés qui, selon elles, dépend des normes subjectives, des attitudes et de l'innovativité cognitive de ces objets. L'innovativité est un trait distinctif des individus qui adoptent de manière précoce les nouveaux produits indépendamment de leur entourage (Rogers, 1962). Parlant de design, Anne Cécile LENOEL s'interroge sur les manières dont l'expertise de ce domaine peut produire et connecter des idées et faire de la participation des publics un facteur de choix (p.121) dans l'appropriation. Avec trois projets exemplaires, elle met en relief les nouvelles logiques de circulation et de partage des données à travers les ambivalences du potentiel économique du phénomène de la conception par participation à partir des objets connectés.

5 La seconde partie, elle aussi composée de sept articles, traite des pratiques sociales des objets connectés sur le plan de la santé, culturel, administratif et agricole. En ce qui concerne l'e-santé, Etienne MOMO propose le déploiement de la capabilité dans la stratégie d'autonomisation et de responsabilisation du sujet dans le paysage des mutualistes de la santé. La capabilité, selon Amartya Sen est la possibilité de choisir et de profiter pleinement des dotations en biens (Sen, $2003: 64$ ). Au sein des organisations culturelles d'Afrique, cette capabilité se manifeste sous la forme de médiation dans la circulation d'information, l'éclaircissement ou le rétablissement des relations. Ainsi, Samuel TIETSE dans ses travaux montre en quoi les utilisateurs des dispositifs de médiation sont des acteurs dans le processus d'innovation des services au sein des structures culturelles. A ce sujet, Kouméalo ANATE et Holonou DANKLOU se servent du concept d'interopérabilité organisationnelle et technique (p.211) et déplorent l'absence de documents pour découvrir, identifier, recueillir et analyser les besoins requis sur le plan technique, réglementaire et politique pour le développement de l'e-gouvernement au Togo. Ainsi, pour ces auteurs, l'interconnexion dans la fourniture des services spécifiques d'administration en ligne et entre les systèmes technologiques apparaît comme un facteur susceptible de limiter l'exclusion dans la mise en place de l'egouvernement au Togo.

6 Enfin, l'ouvrage aborde la contribution des objets connectés à l'amélioration des conditions de travail et de commercialisation agricole. On peut retenir ici que l'utilisation du téléphone mobile au Bénin chez les producteurs de l'ananas dépend des facteurs sociaux tels que le niveau d'étude et le niveau de revenu (Kossi AMESSINOU, 
p. 250). Au Burkina Faso, les maraichéculteurs, utilisateurs du Système d'Information des Marchés de l'Agriculture (SIMAgri), soutenu par le ministère de l'agriculture, peuvent consulter les prix sur les réseaux sociaux, mettre en ligne leurs productions et préciser le jour de disponibilité des produits. Toutefois, l'analphabétisme reste un blocage à l'utilisation des plates-formes web. L'ouvrage vient consolider le regard porté sur l'utilisateur en tant qu'acteur dans le processus de l'innovation. Elle explore surtout les nouvelles pratiques des objets connectés qui se développent au sein des populations en quête de développement «individuel» sur la base de théories, de données empiriques et d'analyse de documents. Les concepts abordés sont susceptibles de susciter d'autres questions de recherche chez le chercheur en Sciences de l'Information et de la Communication et en Sciences Sociales et Humaines qui s'intéresse à l'usage des TIC dans les pays d'Afrique.

\section{AUTEURS}

\section{CAROLE FAGADÉ}

Université Bordeaux Montaigne 